\title{
Co-evolution of Sensors and Controllers
}

\author{
Komei Sugiura* Non-member \\ Takayuki Shiose* Non-member \\ Hiroshi Kawakami* Non-member \\ Osamu Katai* ${ }^{*}$ Member
}

In this paper we investigate the evolutionary development of embodied agents that evolve not only control mechanisms but also the sensitivity and temporal resolution of their sensors. In our system, agents can adapt their sensory parameters to their morphology and task environments, while no explicit cost for sensors is used. We have carried out two kinds of experiments in which agents perform phototactic and pursuit/evading behaviors. The experimental results indicate that the sensors and controller co-evolve in an agent through interaction with the environments.

Keywords: sensor evolution, evolutionary robotics, temporal resolution, embodiment

\section{Introduction}

Artificial Intelligence (AI) has exhibited its ability to solve problems under very limited conditions such as playing chess and solving mathematical problems. In these cases, human programmers have to design limited and digitized input information for the AI. "AI-Robots" themselves are not capable of pick out meaningful inputs from the environment according to the problem to be solved. As a result of this situation, the Frame Problem ${ }^{(1)(2)}$ arises.

How do animals, including humans, cope with this Frame Problem? Uexküll insisted that animals have their own worlds (Umwelten) in which they are surrounded by information that is meaningful to them ${ }^{(3)}$. Namely, animal species have evolved their sensory systems to pick out information that is meaningful only to them, so that each of them 'sees' the world differently.

Animals' behavior and their Umwelten are closely related to each other. The sensitivity and resolution of insects' sensors depend on their feeding behavior, mating behavior, velocity and so on ${ }^{(4)}$. For instance, the human visual system has high spatial resolution and low temporal resolution, whereas the fly visual system has low spatial resolution and high temporal resolution. Critical Fusion Frequency (CFF) is an indicator of temporal resolution. Generally, insects that move at high speed have a high CFF, while insects that move slowly have a low CFF.

The sensitivity and resolution of sensors differ not only among animal species but also in a single species. For example, the fovea/periphery in the visual systems of both humans and mantes have high/low spatial resolution. In this case, the diversification of the ability of sensors is determined by the animals' physical characteristics.

\footnotetext{
* Graduate School of Informatics, Kyoto University

Yoshida-Honmachi, Sakyo-ku, Kyoto 606-8501, Japan
}

Inspired by these biological facts, some AI researchers have tried to artificially evolve the morphology and behavior of an agent in the field of Artificial Life and Evolutionary Robotics ${ }^{(5)}$. Sims and Lipson et al. developed a system that evolves the morphology and neural system of robots inside a computer ${ }^{(6)(7)}$. Other research efforts have been made on Sensor Evolution ${ }^{(8) \sim(13)}$. Kortmann et al. investigated the evolution of spatial and temporal resolution in populations of simulated visuo-motor systems, and showed that two factors are responsible for the trade-off between spatial and temporal resolution ${ }^{(14) \sim(16)}$. However, no previous studies has achieved a system that is able to adapt both its controller and its sensory system, which segmentalise the environment, to its physical characteristics and the task.

In this paper, we investigate the evolution of the sensors and controller of embodied agents. We develop simulated sensory-motor systems that can change the sensitivity and temporal resolution of their sensors. Then, we carry out two kinds of experiments in which populations of the sensory-motor systems perform tasks. Finally, we describe how the physical characteristics of agents affect the diversification of their sensors through interaction with the environment.

\section{Sensory-Motor System and Task Environments}

We constructed sensory-motor systems that can change sensitivity of the sensors, temporal resolution of sensors, and connection weights in their neural network controllers. To investigate how embodied agents adapt their sensors and controllers to the task environment, we prepared two kinds of environments in which populations of sensory-motor systems perform different tasks. The individuals and environments were built using the robot simulator Webots ${ }^{\dagger}$. Below we provide the details

$\dagger$ http://www. cyberbotics.com 

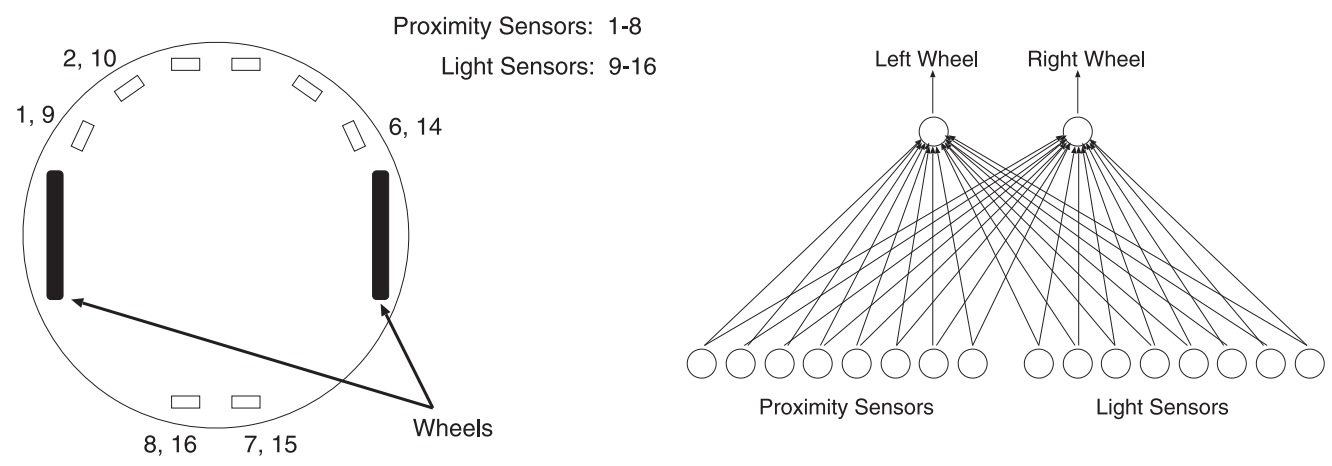

Fig. 1. Left: Top view of the structure of the simulated sensory-motor system (agent). The system is based on the Khepera robot; $28 \mathrm{~mm}$ in radius, and equipped with eight infrared sensors used as both proximity sensors (1-8) and light sensors (9-16). Right: Schematic view of Braitenberg's Vehicle-like controller ${ }^{(17)}$. It has 16 input units, half of which receive information from the proximity sensors and the other half receive information from the light sensors, with two output units controlling the speed of the wheels.

of the sensory-motor system and the task environments.

2.1 Sensory-Motor System A top view of the sensory-motor system is shown in Fig. 1.

\section{Sensors}

This Khepera-like agent has eight infrared sensors that are used as both proximity sensors and light sensors. The two kinds of sensors are physically a single device, but the simulator models it as two different devices by applying different computing algorithms.

Each sensor has various sensitivity and resolution values. Here, the sensitivity $s_{i}$ stands for a radix used to digitize the $i^{t h}$ sensor's input. The bigger $s_{i}$ becomes, the more the sensor is able to detect slight changes.

The temporal resolution $\Delta t_{i}$ stands for the interval that the $i^{t h}$ sensor's input is updated. Consequently, the sensor is unable to detect changes occurring within $\Delta t_{i}$. Therefore, CFF is modeled by $1 / \Delta t_{i}$.

We put a restriction on $s_{i}$ and $\Delta t_{i}$ in order to hold the amount of acquired information $I$ constant. Here, $I$ is defined as information that an agent can acquire from all of its sensors in a unit of time. Thus, $s_{i}$ and $\Delta t_{i}$ must fulfill the following equation.

$$
\sum \frac{s_{i}}{\Delta t_{i}}=I \ldots \ldots \ldots \ldots \ldots \ldots \ldots \ldots \ldots \ldots \ldots \ldots
$$

In our system, $I$ is considered to be resource for sensors, and distributed to every sensors. Note that no explicit cost for sensors is necessary in our system.

\section{Controller}

The system is controlled by a simple neural network, which has only inputs, outputs, and multiplying connections. The right-hand side figure of Fig. 1 shows a schematic view of the controller. The rotation of each wheel is stimulated or inhibited in proportion to the normalized signal strength that each sensor detects. The connection weights are encoded in the individual's genotype, so they are fixed during one generation.

\section{Actuators}

Wheels are driven by two independent motors. The maximum angular speed $\omega_{\max }[\mathrm{rad} / \mathrm{s}]$ can be varied, while the maximum angular acceleration $\alpha_{\max }\left[\mathrm{rad} / \mathrm{s}^{2}\right]$ is fixed in all experiments $\left(\alpha_{\max }=100\right) . \alpha_{\max }$ brings about inertia effect, so that there is time lag between a motor output and the actual speed of the wheel driven by the motor.

2.2 Task Environments We carried out the following two kinds of experiments.

\section{(I) Phototaxis}

One individual is put in an arena $(100 \mathrm{~mm} \times 100 \mathrm{~mm})$ and performs a phototaxis task. Individuals acquire fitness if they arrive at the circle in which a light source exists (see Fig. 2). Each individual is given one trial at each generation. The position and direction of each individual are initialized randomly at the start of each trial. A trial ends either when the individual arrives at the goal or when 300 time steps have been performed. Here, one time step corresponds to $64 \mathrm{~ms}$.

\section{(II) Predator-Prey Pursuit}

Two species of agents (predator and prey) are used in this experiment. Several researchers have investigated predator-prey co-evolution in both simulated ${ }^{(18)(19)}$ and real robotic experiments ${ }^{(20)(21)}$. Cliff and Miller also showed that task-dependent morphological parameters (view range) are evolved in co-evolving predator and prey agents ${ }^{(18)(19)}$. In this experiment, we investigated dependencies on the ecological niche and the sensitivity and resolution of agents' sensors.

The same arena as (I) is used, but there is no light source. Instead, each predator/prey agent is equipped with a light source on it. Both predators and preys are also provided with sensors having the same range.

At each generation, all couples of randomly chosen predator and prey agents perform tournaments. In each tournament, both the predator and the prey are put randomly in the arena. Once the tournament has started, it continues until the individuals collide or 300 time steps have been performed. We examined several cases in which the prey has different $\omega_{\max }$. 

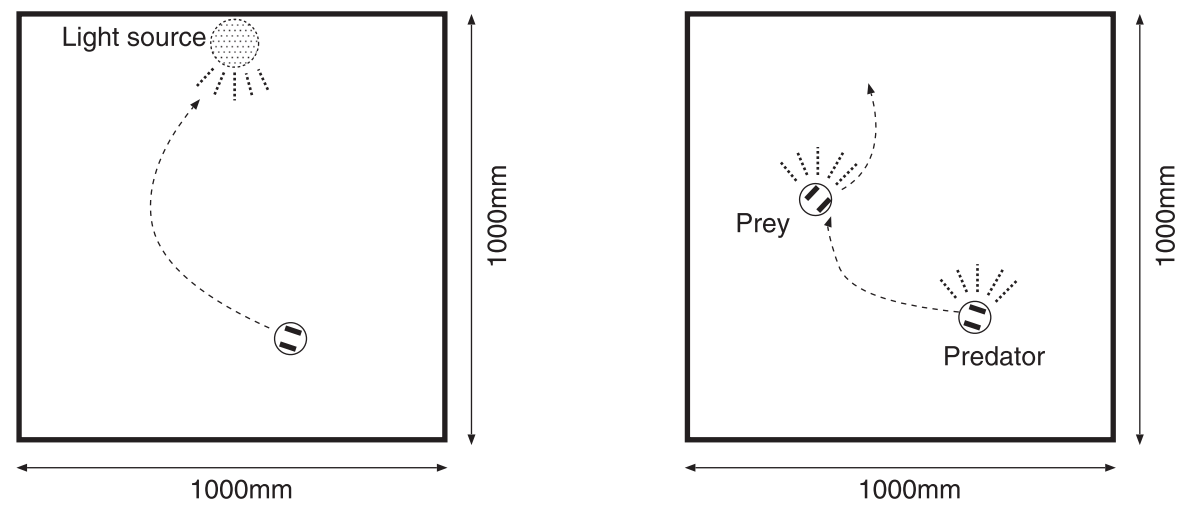

Fig. 2. Left: Schematic view of the arena used in (I). Agents are asked to perform phototactic behavior. Right: Prey evades from the predator in the arena. Sensors equipped on the two species have the same range. A light source is also put on each agent.

\section{Evolution of Sensors and Controllers}

Genetic algorithms (GAs) are used in order to make simulated embodied agents adapt both their sensor properties and behaviors to their morphology and tasks. The GA is allowed to optimize four kinds of parameters of each sensor: sensitivity $s_{i}$, temporal resolution $\Delta t_{i}$, left wheel input multiplier $l_{i}$, and right wheel input multiplier $r_{i}$.

3.1 Genetic Representation The genotype of an individual has the following form:

$$
G=\left(s_{1}^{\prime}, t_{1}^{\prime}, l_{1}, r_{1}, s_{2}^{\prime}, t_{2}^{\prime}, l_{2}, r_{2}, \ldots, s_{16}^{\prime}, t_{16}^{\prime}, l_{16}, r_{16}\right)
$$

where $s_{i}^{\prime}$ and $t_{i}^{\prime}$ are the ratios of spatial and temporal resolution. Each of the parameters $\left(s_{i}^{\prime}, t_{i}^{\prime}, l_{i}, r_{i}\right)$ is encoded into four bits in the genotype. An individual has 16 sensors altogether, therefore the length of a genome is 256 bits.

The weights $\left(l_{i}, r_{i}\right)$ are decoded into real values ranging from -1 to 1 , and $s_{i}$ and $\Delta t_{i}$ are calculated as follows to fulfill the equation (1):

$$
\begin{gathered}
s_{i}=s_{i}^{\prime} \sqrt{\frac{I}{\sum \frac{s_{j}^{\prime}}{t_{j}^{\prime}}}} \\
\Delta t_{i}=t_{i}^{\prime} \sqrt{\frac{\sum \frac{s_{j}^{\prime}}{t_{j}^{\prime}}}{I}}
\end{gathered}
$$

3.2 Genetic Algorithm Each genotype of an individual in the first generation is a random binary string. In each generation, the GA assesses the fitness of all individuals. Roulette selection is used to select individuals that participate in mating. The GA then generates new children from selected individuals to replace the $10 \%$ worst individuals of the population. In creating a new population, the GA uses two types of genetic operators: one-point crossover and point mutation.

Each population consists of 100 individuals in (I). In (II), 100 predators and 100 preys compose independent populations. We used one-point crossover and point mutation as genetic operations. We made the crossover rate $50 \%$ and the probability of mutation per bit $0.2 \%$.
3.3 Fitness Function The fitness function $\Phi$ for individuals in (I) is calculated as follows:

$$
\Phi=1-\frac{t}{T_{\max }}
$$

where $t$ is the time to arrive at the goal and $T_{\max }$ is the maximum time steps performed in the experiment.

For (II), we adopted a fitness function similar to that of ${ }^{(20)}$. Namely, both $\Phi_{p r}$ for a predator and $\Phi_{p y}$ for a prey are based on the time to contact:

$$
\begin{aligned}
& \Phi_{p r}=1-\frac{t}{T_{\max }} \\
& \Phi_{p y}=\frac{t}{T_{\max }}
\end{aligned}
$$

where $t$ is the time the predator needs to contact the prey.

\section{Results and Discussion}

4.1 Performance We carried out experiments in five populations for both (I) and (II). We kept $I=32$ and $\alpha_{\max }=100$. The factor $\omega_{\max }$ varied between the populations, but was kept fixed within a population in (I). In the experiments (II), we kept the predators' $\omega_{\max }=60$, but varied the preys' $\omega_{\max }^{\prime}$ between the prey populations.

The average fitness of the individuals of (I) is plotted against the number of generations in the left-hand side figure of Fig. 3, and the average fitness of the predators of (II) is plotted against the number of generations in the right-hand side figure of Fig. 3. Fig. 3 shows that all populations converged within 300 generations in (I), and the predators' fitness values also converged within 300 generations except one case $\left(\omega_{\max }=20\right)$ in (II).

The right-hand side figure of Fig. 3 shows that the fitness of the predators (preys' angular speed $\omega_{\max }^{\prime}=$ 100) reaches higher values than that of the predators $\left(\omega_{\max }^{\prime}=20\right)$. This is because quick preys hit the wall frequently and are captured by predators easily. Therefore this result can be explained by the fact that $I$ and the range of the sensors are not enough for the preys with high $\omega_{\max }^{\prime}$ to avoid the wall.

4.2 Sensory Specializations We also investi- 
gated the variation of the percentage of information acquired from each sensor. Here, the percentage $r_{i}$ from $i^{t h}$ sensor is calculated as follows:

$$
r_{i}=\frac{\frac{s_{i}}{\Delta t_{i}}}{I} \times 100
$$

In Fig. 4, the average $r_{i}$ within a population of agents in (I) is plotted against the number of generations. The left-hand side figure shows that $r_{i}$ does not vary widely within sensors after 300 generations. This indicates that the agents after 300 generations have adapted their sensory parameters so that they can acquire information from both proximity sensors and light sensors. On the other hand, the agents with low $\omega_{\max }$ acquire almost all information from one light sensor (see the left-hand side figure of Fig. 4). This result can be explained by the fact that agents with high $\omega_{\max }$ need more information from the proximity sensors so as not to hit the wall.

In Fig. 5, the average $r_{i}$ within a population of predators in (II) is plotted against the number of generations. In the left-hand side figure of Fig. $5, r_{i}$ is biased toward light sensors compared with the right-hand side figure. This means that the predators after 300 generations have evolved their sensory parameters to concentrate attention on two light sensors to catch the relatively quick preys. Fig. 3 and Fig. 5 show that the fitness of the predators drastically increases with the increase in $r_{i}$ of light sensors. This clearly indicates that sensors and controllers have evolved in agents, so that they have adapted themselves to the task environments.

\section{Conclusions}

In this paper, we investigated the dependencies on the behaviors and morphology in populations of sensorymotor systems. Genetic algorithms (GAs) were used to make simulated embodied agents adapt both their sensor properties and behaviors to their morphology and tasks. We carried out two kinds of experiments in which agents were to perform phototactic and pursuit/evading behaviors. The experimental results show that the physical characteristics of an agent and the task environment affect the sensitivities and the resolution of its sensors, and thus task-dependent morphology are evolved. Future work will include the evolution of sensor morphology (placement, resolution, sensitivity, etc.) in agents that perform tasks in noisy environments.

(Manuscript received December 24, 2003, revised June $4,2004)$

\section{References}

(1) J. McCarthy and P. J. Hayes: "Some philosophical problems from the standpoint of artificial intelligence", Machine Intelligence 4 (Eds. by B. Meltzer and D. Michie), Edinburgh University Press, pp.463-502 (1969)

(2) D. C. Dennett: "Cognitive wheels: The frame problem of AI", Minds, Machines and Evolution: Philosophical Studies (Ed. by C. Hookaway), Cambridge University Press, Cambridge, pp.129-151 (1984)

(3) J. von Uexküll: "A stroll through the worlds of animals and men", Instinctive Behavior: The Development of a Modern Concept (Ed. by C. H. Shiller), International University Press, pp.5-80 (1957)

(4) D. B. Dusenbery: "Sensory ecology: how organisms acquire and respond to information", W. H. Freeman \& Co. (1992)

(5) S. Nolfi and D. Floreano: "Evolutionary Robotics: The Biology, Intelligence, and Technology of Self-Organizing Machines", MIT Press, Cambridge, MA. (2000)

(6) K. Sims: "Evolving 3D morphology and behavior by competition", Artificial Life IV: Proceedings of the Fourth International Workshop on the Synthesis and Simulation of Living Systems (Eds. by R. Brooks and P. Maes), Cambridge, MA., MIT Press, pp.28-39 (1994)

(7) H. Lipson and J. B. Pollack: "Automatic design and manufacture of robotic lifeforms", Nature, 406, 6799, pp.974-978 (2000)

(8) K. Dautenhahn, D. Polani, and T. Uthmann: "Special issue on sensor evolution", Artificial Life, Vol.7, No.2, pp.95-98 (2001)

(9) J. Vaario and K. Shimohara: "On formation of structures", Advances in Artificial Life: Proceedings of the Third European Conference on Artificial Life (Eds. by F. Morán, A. Moreno, J. J. Merelo and P. Chacón), Berlin, Springer Verlag, pp.421-435 (1995)

(10) A. Mark, D. Polani, and T. Uthmann: "A framework for sensor evolution in a population of Braitenberg vehicle-like agents", Artificial Life VI: Proceedings of the Sixth International Conference on Artificial Life (Eds. by C. Adami, R. Belew, H. Kitano and C. Taylor), Cambridge, MA., MIT Press, pp.428-432 (1998)

(11) L. Lightensteiger and R. Pfeifer: "An optimal sensor morphology improves adaptability of neural network controllers", ICANN (Ed. by J. R. Dorronsoro), Springer, pp.850-855 (2002)

(12) K. Balakrishnan and V. Honavar: "On sensor evolution in robotics", Proceedings of the First International Conference on Genetic Programming, Stanford University, CA., pp.455460 (1996)

(13) H. H. Lund, J. Hallam and W.P. Lee: "Evolving robot morphology", Proc. of IEEE 4th International Conference on Evolutionary Computation, IEEE Press, p. Invited paper (1997)

(14) R. Kortmann, E. Postma, and J. van den Herik: "The tradeoff between spatial and temporal resolution in visual systems", Proc. of the eleventh Belgian-Dutch conference on artificial intelligence (Eds. by E. O. Postma and M. Gyssens), pp.219226 (1999)

(15) R. Kortmann, E. Postma, and J. van den Herik: "Visual resolution evolving to a trade-off curve", From animals to animats 6 , proceedings of the sixth international conference on simulation of adaptive behavior (Eds. by J.-A. Meyer, A. Berthoz, D. Floreano, H. Roitblat and S. Wilson), Cambridge, MA., MIT Press, pp.405 - 412 (2000)

(16) R. Kortmann, E. Postma, and J. van den Herik: "Evolution of visual resolution constrained by a trade-off", Artificial Life, 7,2 , pp. $125-145(2001)$

(17) V. Braitenberg: "Vehicles: Experiments in Synthetic Psychology", MIT Press, Cambridge, MA. (1984)

(18) D. Cliff and G. F. Miller: "Tracking the red queen: Measurements of adaptive progress in co-evolutionary simulations", Advances in Artificial Life: Proceedings of the Third European Conference on Artificial Life (Eds. by F. Morán, A. Moreno, J. J. Merelo and P. Chacón), Berlin, Springer Verlag, pp.200-218 (1995)

(19) D. Cliff and G. F. Miller: "Co-evolution of pursuit and evasion II: Simulation methods and results", From animals to animats 4 (Eds. by P. Maes, M. J. Mataric, J.-A. Meyer, J. B. Pollack and S. W. Wilson), Cambridge, MA., MIT Press, pp.506-515 (1996)

(20) D. Floreano, S. Nolfi, and F. Mondada: "Competitive coevolutionary robotics: From theory to practice", Proceedings of the Fifth International Conference of the Society for Adaptive Behavior (Eds. by R. Pfeifer, B. Blumberg and H. Kobayashi), Cambridge, MA., MIT Press, pp.515-524 (1998)

(21) S. Nolfi and D. Floreano: "Co-evolving predator and prey robots: Do 'arms races' arise in artificial evolution?", Artificial Life, Vol.4, No.4, pp.311-335 (1999) 

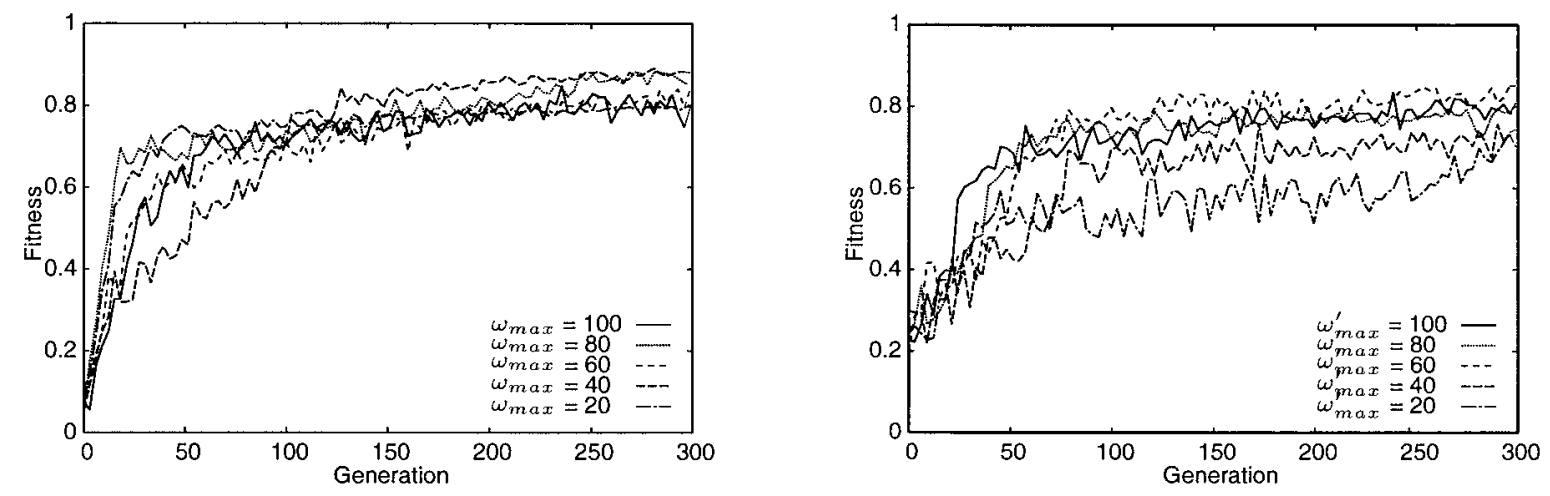

Fig. 3. Left: Results of Experiment (I). Each curve represents the average fitness of the five different populations. Right: Results of Experiment (II). Each curve represents the average fitness of the five different populations of predators.
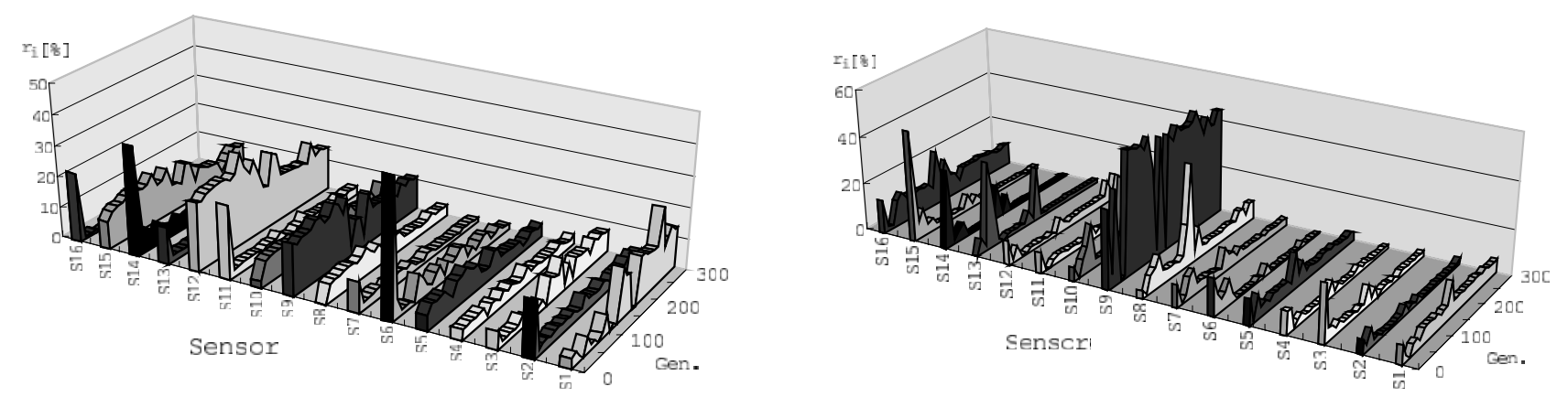

Fig. 4. The variation of the average percentage of information acquired from each sensor within a population of agents in (I). Each of $\{\mathrm{S} 1, \ldots, \mathrm{S} 16\}$ stands for each of sensors. Left: $\omega_{\max }=100$. Right: $\omega_{\max }=20$.
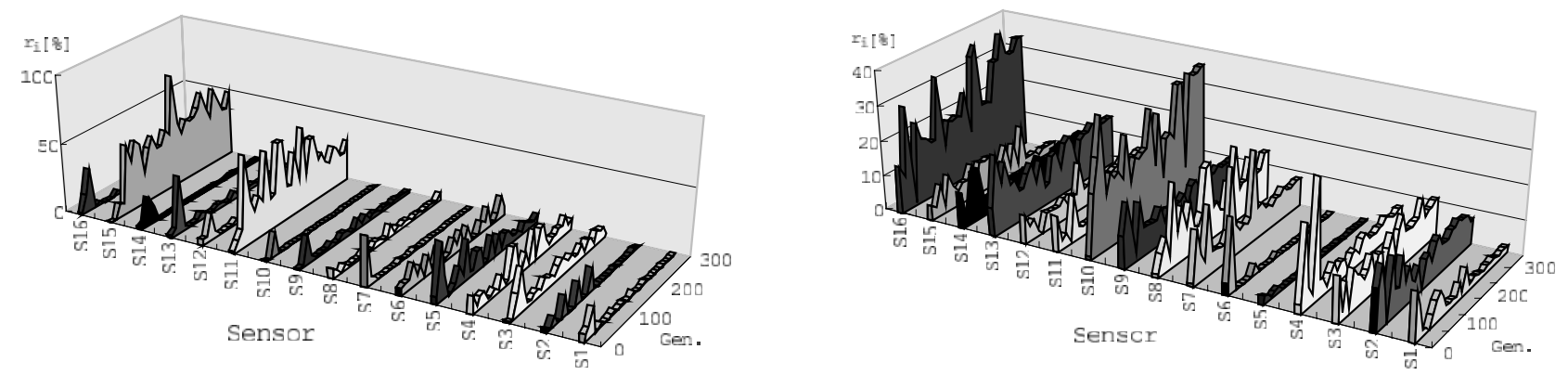

Fig. 5. The variation of the average percentage of information acquired from each sensor within a population of predators in (II). Left: The predators' $\omega_{\max }=60$, and the preys' $\omega_{\max }^{\prime}=100$. Right: The predators' $\omega_{\max }=60$, and the preys' $\omega_{\max }^{\prime}=20$. 
Komei Sugiura (Non-member) is a Ph.D. student at the

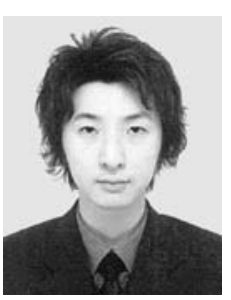
Department of Systems Science, Graduate School of Informatics, Kyoto University. He received his B.S. and M.S. degrees from Kyoto University in 2002 and 2004, respectively. His current research interests include evolutionary robotics and artificial life. He is a member of the Society of Instrument and Control Engineers, and the Japanese Society for Artificial Intelligence.

Takayuki Shiose (Non-member) is a research associate at

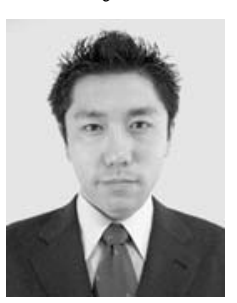
the Department of Systems Science, Graduate School of Informatics, Kyoto University, and also a visiting researcher at ATR Human Information Science Labs. He received his B.S., M.S., and Ph.D. degrees in precision engineering from Kyoto University in 1996, 1998, and 2003, respectively. From 1998 to 2000, he was a research fellow of the Japan Society for the Promotion of Science. From 2000 to 2002, he was a research associate at Graduate School of Science and Technology, Kobe University. He is a member of the Society of Instrument and Control Engineers, and the Japan Society of Mechanical Engineers.

Hiroshi Kawakami (Non-member) is an associate professor

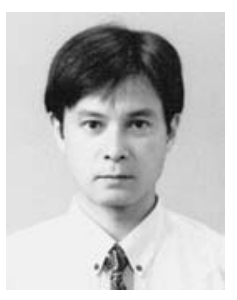
at the Department of Systems Science, Graduate School of Informatics, Kyoto University, Japan. He received his B.S., M.S., and Ph.D. degrees in precision engineering from Kyoto University in 1987, 1989, and 1993, respectively. From 1989 to 1998, he was an instructor at the Department of Information Technology, Faculty of Engineering, Okayama University, and in 1998 he was with the current department as an associate professor. He has been engaged in research on ecological and emergent system design, methodology of collaborative synthesis, human-system communication, and knowledge information processing. He is a member of the Society of Instrument and Control Engineers, Human Interface Society, and the Japanese Society for Artificial Intelligence.
Osamu Katai (Member) graduated from Kyoto University

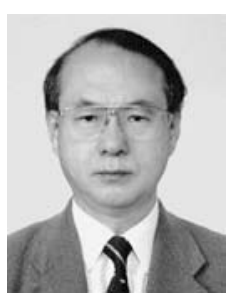
at the Department of Mechanical Engineering in 1969, then proceeded to the Graduate School of Engineering and received M.S. and Ph.D. degrees in 1971 and 1979, respectively. Since 1971, he has been with Kyoto University, first as an Instructor at the Department of Precision Engineering and now is a Professor at the Department of Systems Science in the Graduate School of Informatics. From 1980 to 1981, he was a visiting researcher at INRIA (National Research Institute on Informatics and Automation, France). His current research interests are on the methodologies and theoretical frameworks of 'symbiotic systems' where artificial systems and natural coexist harmoniously. He is a member of the Japanese Society for Artificial Intelligence and the Society of Instrument and Control Engineers, as well as a number of other scientific societies. 